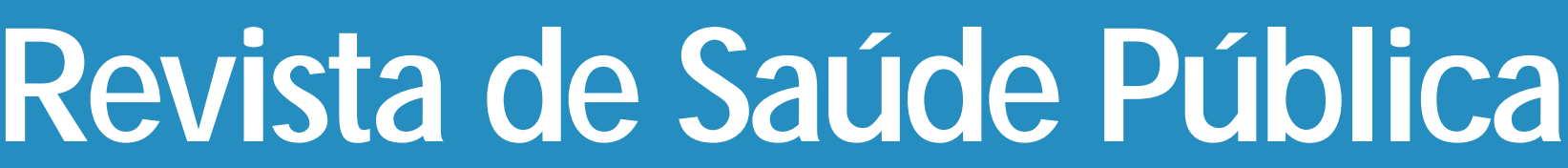

$\begin{array}{llllll}\mathbf{O} & \mathbf{U} & \mathbf{R} & \mathbf{N} & \mathbf{A} & \mathbf{L}\end{array}$

0 F

$\mathbf{P} \mathbf{U}$

B L I C

H E A $\quad$ L $\quad$ T

\title{
Variações regionais e interespecíficas na morfologia de insetos do complexo Lutzomyia intermedia (D iptera, Psychodidae, Phlebotominae)
}

Regional and interspecific variations in the morphology of insects of the Lutzomyia intermedia complex (Diptera, Psychodidae, Phlebotominae)

Carlos B. Marcondes, Ana L. Lozovei e Eunice A. B. G alati

Departamento de Microbiologia e Parasitologia do Centro de Ciências Biológicas da Universidade Federal de Santa Catarina. Florianópolis, SC-Brasil (C.B.M.); Departamento de Patologia Básica do Setor de Ciências Biológicas da Universidade Federal do Paraná. Curitiba, PR-Brasil (A.L.L.);

Departamento de Epidemiologia da Faculdade de Saúde Pública da U niversidade de São Paulo.

São Paulo, SP-Brasil (E.A.B.G.)

MARCO NDES Carlos B., Ana L. Lozovei e Eunice A. B. G alati Variações regionais e interespecíficas na morfologia de insetos do complexo Lutzomyia intermedia (D iptera, Psychodidae, Phlebotominae)

Rev. Saúde Pública, 32 (6): 519-25, 1998

(c) Copyright Faculdade de Saúde Pública da USP. Proibida a reprodução mesmo que parcial sem a devida autorização do Editor Científico. Proibida a utilização de matérias para fins comerciais. All rights reserved. 


\title{
Variações regionais e interespecíficas na morfologia de insetos do complexo Lutzomyia intermedia (Diptera, Psychodidae, Phlebotominae)
}

\author{
Regional and interspecific variations in the morphology of insects \\ of the Lutzomyia intermedia complex \\ (D iptera, Psychodidae, Phlebotominae)
}

\author{
Carlos B. Marcondes, Ana L. Lozovei e Eunice A. B. G alati \\ Departamento de Microbiologia e Parasitologia do Centro de Ciências Biológicas da Universidade \\ Federal de Santa Catarina. Florianópolis, SC-Brasil (C.B.M.); Departamento de Patologia Básica do \\ Setor de Ciências Biológicas da Universidade Federal do Paraná. Curitiba, PR-Brasil (A.L.L.); \\ Departamento de Epidemiologia da Faculdade de Saúde Pública da Universidade de São Paulo. \\ São Paulo, SP-Brasil (E.A.B.G.)
}

\begin{abstract}
Resumo
Introdução

Lutzomyia intermedia s. s. e L. neivai, usualmente consideradas como pertencentes a uma só espécie, constituem um complexo de espécies. Foram analisadas as medidas de várias estruturas de exemplares das duas espécies, provenientes do Brasil, Paraguai, Argentina e Bolívia.

Método Foram medidas 39 estruturas em exemplares de ambos os sexos, com ocular graduada, fazendo-se comparações por análise de variância (ANOVA).

Resultados

Constatou-se desvio significativo nas proporções de fêmeas e de machos com cada fórmula palpal e influência da região de origem dos insetos. O labro e os palpos maxilares foram mais longos nas fêmeas e o antenômero III mais longo nos machos. Foram constatadas várias diferenças entre medidas das asas, quase todas maiores nas fêmeas. A proporção de espermatecas com cabeça simples em L. neivai é significativamente maior que em L. intermedia. Também foram observadas diferenças significativas nos comprimentos das bombas e dos dutos ejaculadores entre as espécies.
\end{abstract}

Discussão

\begin{abstract}
As variações nas fórmulas palpais ressaltam o risco do uso desta fórmula para a associação entre exemplares de ambos os sexos. As diferenças nos comprimentos dos palpos e no labro podem estar ligadas à hematofagia das fêmeas. Comenta-se sobre as possíveis implicações da maior relação comprimento/largura das asas em machos. As diferenças nas proporções de fêmeas das duas espécies com os diferentes tipos de cabeça de espermatecas podem auxiliar na identificação específica. As diferenças nos comprimentos das bombas e dos dutos ejaculadores e nas relações entre estes comprimentos podem auxiliar na identificação dos machos, ainda difícil.
\end{abstract}

Psychodidae, anatomia e histologia. Leishmaniose mucocutânea. Morfometria.

Correspondência para/Correspondence to: Carlos Brisola Marcondes - Campus Trindade - 88040-900 Florianópolis, SC - Brasil. E- mail:cbrisola@mbox1.ufsc.br.

Edição subvencionada pela FAPESP (Processo n 97/09815-2).

Recebido em 16.12.1997. Reapresentado em 18.5.1998. Aprovado em 9.6.1998. 


\begin{abstract}
Introduction

Lutzomyia intermedia s. s. and L. neivai, usually considered as belonging to just one species, constitute a complex of species. The measurements of several anatomical structures of specimens of both groups, from Brazil, Paraguay, Argentina and Bolivia were analysed.

Method Thirty-nine structures were measured in specimens of both sexes, using a graduated ocular, analysing comparisons by analyses of variance (ANOVA).

Results A significant deviation in the proportions of females and males showing each palpal formula and the influence of the region of origin of the flies were verified. Labrum and maxilary palps were longer in females and Antennomere III was longer of males. Several significant differences in the measurements of wings were noted, most of them greater in females. The proportion of spermathecae with simple heads was significantly greater in L. neivai than in $L$. intermedia. Significant differences in the length of genital pump and filaments between the species were also noted.

Discussion The variation in the palpal formulae in both sexes show the risk of the use of this formula for the association between specimens of the two sexes. The differences in the lengths of palps and labrum between the sexes could be related to female blood feeding. The possible biological significance of the sexual differential relation length/width of wings is commented on. The different proportions of females of the two species with each spermathecae head shape may help in identification. The differences in the lengths of genital pumps and filaments and the relation between them could help in the identification of males, which is still difficult.
\end{abstract}

Psychodidae, anatomy. Leishmaniasis, mucacutaneous. Morphometry.

\section{INTRO DU ÇÃO}

Marcondes ${ }^{13}$ (1996) redescreveu L. neivai (Pinto, 1926) e propôs a sua revalidação, dis-tinguindo esta espécie de $L$. intermedia $s$. $s$. Para a distinção das fêmeas de ambas, foram utilizadas várias características morfológicas, especialmente nas espermatecas e seus dutos e nos dentes horizontais do cibário, para a distinção entre as fêmeas das duas espécies. A distinção entre os machos ainda é considerada impossível com análise univariada*, apesar de algumas variações nas dimensões de certas estruturas.

Marcondes e col. ${ }^{14}$ comentaram sobre as várias referências ao possível papel vetorial de membros do complexo L. intermedia (L. intermedia e L. neivai), bem como sobre a distribuição geográfica de ambos.

Além das características anteriormente utilizadas na distinção das duas espécies, foram estudadas variações morfológicas inter e intra-específicas de várias estruturas, relacionadas com a origem dos espécimes. Estas variações podem ser úteis para a identificação específica, bem como na análise das influências do clima, e se constituem no objeto do presente trabalho.

\section{MÉTO DO}

Os insetos estudados foram montados em líquido de Berlese ou em Enecê. Foram medidas 39 estruturas da cabeça, tórax e abdome de exemplares de ambos os sexos de $L$. intermedia e L. neivai. Os machos só foram identificados como de uma ou outra espécie se associados a fêmeas corretamente identificadas, da mesma procedência.

Os dados foram analisados por análise de variância (ANOVA), com o auxílio de programa Excel 4.0. As variáveis independentes estabelecidas foram as regiões e os sexos dos insetos, e as dependentes foram as medidas de estruturas ou relações entre medidas. A precisão experimental foi calculada pela fórmula $100 . \mathrm{s} / \mathrm{m}$ (s- desvio-padrão; m- média). A análise de significância de diferenças nas frequiências de certas características foi feita por $\mathrm{X}^{2}$.

As quantidades observadas nas tabelas de frequiências foram transformadas em percentagens dos totais, às quais foi acrescentado 0,5 , obtendo-se a seguir a raiz quadrada

$(\sqrt{\%+0,5})$, para fazer um delineamento de blocos casualizados, comparando-se o $\mathrm{F}$ assim obtido com o $\mathrm{F}_{\text {tab }}$. Os insetos de cada região constituíram os blocos casualizados.

*Análise realizada com Rede Neural Artificial permitiu a distinção dos machos das duas espécies (Marcondes e col., dados não publicados) 


\section{RESU LTAD O S}

As Tabelas 1 e 2 mostram, respectivamente para fêmeas e machos, as quantidades de insetos com cada fórmula palpal, separados por espécies e por região de origem. Os valores de $c^{2}$ indicam que há correlação entre a região de origem e a freqüência das fórmulas palpais, e os valores de $\mathrm{F}$ referem-se a diferenças significativas entre as fórmulas.

A fórmula 1.4.5.2.3 é muito mais freqüente que

Tabela 1- Q uantidade de fêmeas de L. intermedia e de $L$. neivai estudadas, agrupadas de acordo com a região de origem e a fórmula palpal.

\begin{tabular}{|c|c|c|c|c|c|c|c|c|c|}
\hline \multirow{2}{*}{ FP } & \multicolumn{4}{|c|}{ L. intermedia } & \multicolumn{4}{|c|}{ L. neivai } & \multirow{2}{*}{ Total } \\
\hline & $N E+M E$ & ES & RJ & $\mathrm{SL}$ & $\mathrm{GO}+\mathrm{MW}$ & $\mathrm{SI}$ & R. Sul & Outros & \\
\hline 1.4 .5 .2 .3 & 23 & 48 & 24 & 24 & 2 & 54 & 11 & 26 & 212 \\
\hline 1.4.2.5.3 & 3 & 11 & 6 & 7 & - & 12 & 3 & 3 & 45 \\
\hline 1.4 .2 .3 .5 & - & - & - & 2 & - & - & - & 1 & 3 \\
\hline 1.4 .3 .2 .5 & - & - & - & - & 1 & 2 & - & - & 3 \\
\hline 1.4.5.3.2 & 2 & - & - & - & - & 1 & - & - & 3 \\
\hline 1.4.5.(2.3) & - & - & 1 & - & - & 1 & - & - & 2 \\
\hline 1.4.(2.5).3 & - & - & 1 & - & - & - & - & - & 1 \\
\hline Total & 28 & 59 & 32 & 33 & 3 & 70 & 14 & 30 & 269 \\
\hline
\end{tabular}

Tabela 2 - Q uantidade de machos de L. intermedia e de L. neivai estudados, agrupados de acordo com a região de origem e a fórmula palpal.

\begin{tabular}{|c|c|c|c|c|c|c|c|c|c|}
\hline \multirow{2}{*}{ FP } & \multicolumn{4}{|c|}{ L. intermedia } & \multicolumn{4}{|c|}{ L. neivai } & \multirow{2}{*}{ Total } \\
\hline & $\mathrm{NE}+\mathrm{ME}$ & ES & $\mathrm{RJ}$ & $\mathrm{SL}$ & $\mathrm{GO}+\mathrm{MW}$ & $\mathrm{SI}$ & R. Sul & Outros & \\
\hline 1.4 .5 .2 .3 & 9 & 2 & 5 & 2 & - & - & - & - & 18 \\
\hline 1.4 .2 .5 .3 & 13 & 20 & 17 & 14 & 2 & 32 & 7 & 17 & 122 \\
\hline 1.4.2.3.5 & 8 & 17 & 22 & 10 & 1 & 22 & 6 & 11 & 97 \\
\hline 1.4.3.2.5 & - & - & 1 & 1 & - & - & - & - & 2 \\
\hline 1.4.5.3.2 & 1 & - & 1 & - & - & - & - & - & 2 \\
\hline 1.4.5.(2.3) & - & - & - & 1 & - & - & - & - & 1 \\
\hline 1.4.2.(3.5) & 3 & 4 & 6 & 3 & - & 3 & - & 1 & 20 \\
\hline 1.4.(2.5). 3 & 2 & - & 1 & - & - & 2 & - & 1 & 6 \\
\hline Total & 36 & 43 & 53 & 31 & 3 & 59 & 13 & 30 & 268 \\
\hline
\end{tabular}

Tabela 3 - Comparação entre os comprimentos do labro, total dos palpos e do antenômero III de machos e fêmeas de $L$. intermedia e de L. neivai*.

\begin{tabular}{|c|c|c|c|c|c|c|c|c|}
\hline \multirow{2}{*}{ Estrutura } & \multicolumn{4}{|c|}{ Fêmeas } & \multicolumn{4}{|c|}{ Machos } \\
\hline & Média & DP & $\mathrm{N}$ & $\mathrm{CV}$ & Média & DP & $\mathrm{N}$ & $\mathrm{CV}$ \\
\hline \multicolumn{9}{|l|}{ L. intermedia } \\
\hline Compr. Iabro & 339 & 22,33 & 176 & 6,59 & 226 & 13,56 & 188 & 6 \\
\hline Comp. total palpo & 579 & 35,02 & 147 & 6,05 & 458 & 33,93 & 168 & 7,41 \\
\hline Compr. Ant. III & 228 & 19,37 & 163 & 8,51 & 237 & 20,31 & 186 & 8,57 \\
\hline $\begin{array}{l}\text { Ant. III/ comp. labro } \\
\text { L. neivai }\end{array}$ & 0,675 & 0,052 & 153 & 7,64 & 1,05 & 0,086 & 174 & 8,24 \\
\hline Compr. Iabro & 347,1 & 21,88 & 144 & 6,3 & 231 & 14,56 & 131 & 6,29 \\
\hline Comp. total palpo & 587 & 36,11 & 113 & 6,15 & 473 & 28,81 & 106 & 6,09 \\
\hline Compr. Ant. III & 224 & 16,72 & 127 & 7,47 & 241 & 17,15 & 122 & 7,12 \\
\hline Ant. III/ comp. Iabro & 0,647 & 0,049 & 127 & 7,59 & 1,044 & 0,073 & 116 & 7,03 \\
\hline
\end{tabular}

DP- desvio-padrão; N - número de exemplares examinados; CV- coeficiente de variação; Ant. III- antenômero III.

*Significância de todas as comparações (AN OVA)-1\%

ANOVA - análise de variância 
o esperado nas fêmeas, assim como as fórmulas 1.4.2.5.3 e 1.4.2.3.5 o são nos machos. O mesmo padrão repetiu-se para as duas espécies.

Na Tabela 3, estão relacionados os comprimentos de várias estruturas da cabeça de exemplares de ambos os sexos e espécies, com diferenças significativas.

As Tabelas 4 e 5 mostram as medidas de machos e fêmeas, respectivamente, de L. intermedia de Venda Nova do Imigrante (Espírito Santo) e de L. neivai do Interior do Estado de São Paulo.

Todas as medidas alares significativamente diferentes foram maiores nas fêmeas, com exceção da proporção entre o comprimento e a largura máxima da asa.

As quantidades de exemplares com cabeça simples, bilobada, trilobada e truncada/assimétrica foram, respectivamente, $37,95,7$ e 26 para $L$. intermedia s. s. e $125,4,0$ e 3 para $L$. neivai $\left(X^{2}=154,9 ; X_{\text {tab 5\%,7 g.1. }}^{2}=14,07 ;\right.$ F- não significativo $)$.
A Tabela 6 mostra os comprimentos das bombas ejaculadoras, dos dutos ejaculadores e as relações dutos/bombas, para exemplares de ambas as espécies. Os exemplares das quatro primeiras linhas de cada estrutura ou proporção dentro da tabela foram identificados como $L$. intermedia e os das quatro últimas como L. neivai.

\section{DISCUSSÃO}

As variações nas proporções das fórmulas palpais entre machos e fêmeas, em ambas as espécies, indicam que se deve ter cuidado na sua utilização para a associação entre sexos de espécies desconhecidas. $\mathrm{O}$ fato de ter sido encontrada predominância das mesmas fórmulas palpais em cada sexo, para $L$. intermedia e L. neivai, ressalta a semelhança entre ambas as espécies. Ainfluência da região de origem dos flebotomíneos nas proporções das fórmulas palpais podem ter relação com características climá-

Tabela 4 - Comparação entre as medidas (em $\mathrm{mm}$ ) de dimensões das asas de fêmeas e machos de L. intermedia de Venda Nova do Imigrante, Estado do Espírito Santo, Brasil *.

\begin{tabular}{|c|c|c|c|c|c|c|c|c|}
\hline \multirow{2}{*}{ Estrutura } & \multicolumn{4}{|c|}{ Fêmeas } & \multicolumn{4}{|c|}{ Machos } \\
\hline & Média & D P & $\mathrm{N}$ & $\mathrm{CV}$ & Média & DP & $\mathrm{N}$ & $\mathrm{CV}$ \\
\hline Compr. asa & $2.126,0$ & 92,3 & 35 & 4,34 & $1.877,0$ & 68,1 & 29 & 3,63 \\
\hline Larg. asa & 661,3 & 23,39 & 35 & 3,54 & 549,2 & 26,98 & 29 & 4,91 \\
\hline Compr. asa/larg. asa & 3,22 & 0,153 & 34 & 4,75 & 3,42 & 0,145 & 29 & 4,24 \\
\hline$\alpha$ & 632,3 & 43,07 & 37 & 6,81 & 511,4 & 33,53 & 27 & 6,56 \\
\hline$\beta$ & 315,1 & 34,18 & 37 & 10,8 & 286,7 & 26,58 & 28 & 9,27 \\
\hline$\gamma$ & 220,5 & 29,53 & 36 & 13,4 & 152,2 & 32,93 & 26 & 21,6 \\
\hline$\delta$ & 336,9 & 39,19 & 37 & 11,6 & 260,8 & 38,62 & 27 & 14,8 \\
\hline$\alpha / \beta$ & 2,03 & 0,29 & 37 & 14,3 & 1,8 & 0,23 & 27 & 12,8 \\
\hline$\varepsilon$ & 773,4 & 41,67 & 37 & 5,38 & 628,5 & 39,72 & 27 & 6,32 \\
\hline$\delta /$ compr. asa & 0,158 & 0,02 & 35 & 12,6 & 0,138 & 0,02 & 27 & 14,5 \\
\hline Compr. $\mathrm{R}_{5}$ & $1.434,0$ & 46,59 & 37 & 3,25 & $1.253,0$ & 52,49 & 27 & 4,19 \\
\hline
\end{tabular}

DP - desvio-padrão; $\mathrm{N}$ - número de exemplares medidos

$\alpha$ - comprimento de $\mathrm{R}_{2} ; \beta$ - comprimento de $\mathrm{R}_{2+3} ; \gamma$ - comprimento de $\mathrm{R}_{2+3+4} ; \delta$ - distância entre a bifurcação de $\mathrm{R}_{2+3}$ e a extr. distal de $\mathrm{R}_{1} ; \varepsilon$ - comprimento de $\mathrm{R}_{3}$. * Significância de todas as comparações (ANOVA) - $1 \%$

AN OVA - análise de variância

Tabela 5 - Comparação entre as medidas (em $\mathrm{mm}$ ) de dimensões das asas de fêmeas e machos de L. neivai do Estado de São Paulo*.

\begin{tabular}{|c|c|c|c|c|c|c|c|c|}
\hline \multirow{2}{*}{ Estrutura } & \multicolumn{4}{|c|}{ Fêmeas } & \multicolumn{4}{|c|}{ Machos } \\
\hline & Média & $\overline{D P}$ & $\mathrm{~N}$ & $\mathrm{CV}$ & Média & $\mathrm{DP}$ & $\mathrm{N}$ & $\mathrm{CV}$ \\
\hline Compr. asa & $2.086,0$ & 111,7 & 25 & 5,64 & $1.829,0$ & 95,24 & 26 & $\overline{5,21}$ \\
\hline Larg. asa & 644,6 & 49,05 & 23 & 7,61 & 532,7 & 34,36 & 26 & 6,45 \\
\hline$\alpha$ & 616,7 & 57,23 & 27 & 9,28 & 534,6 & 50,76 & 25 & 9,49 \\
\hline$\gamma$ & 215,5 & 29,25 & 25 & 13,6 & 191,1 & 26,15 & 24 & 13,7 \\
\hline$\delta$ & 328,6 & 48,3 & 27 & 14,7 & 278,2 & 34,75 & 25 & 12,49 \\
\hline$\varepsilon$ & 755,7 & 62,95 & 27 & 8,33 & 627,7 & 42,96 & 25 & 6,84 \\
\hline Compr. $\mathrm{R}_{5}$ & $1.394,0$ & 104,8 & 25 & 7,52 & $1.241,0$ & 73,43 & 19 & 5,92 \\
\hline Compr. Iarg. asa & 3,25 & 0,162 & 23 & 4,98 & 3,44 & 0,158 & 26 & 4,59 \\
\hline
\end{tabular}

DP - desvio-padrão; $\mathrm{N}$ - número de exemplares medidos; CV - coeficiente de variação;

$\alpha$ - comprimento de $\mathrm{R}_{2} ; \gamma$-comprimento de $\mathrm{R}_{2+3+4} ; \delta$ - distância entre a bifurcação de $\mathrm{R}_{2+3}$ e a extr. distal de $\mathrm{R}_{1} ; \varepsilon$ - comprimento de $\mathrm{R}_{3}$

* Significância de todas as comparações (ANOVA) - 1\%

ANOVA - análise de variância 
ticas. Espécies dos subgêneros Nyssomyia e Trichophoromyia, em que os artículos palpais 2, 3 e 5 têm comprimentos semelhantes, têm mais tendência a apresentar variações nas fórmulas palpais que os de outros subgêneros, em que estes artículos são bem diferentes.

Abonnenc $^{1}$ (1972) utilizou a fórmula palpal em chaves dicotômicas para a distinção de várias espécies de flebotomíneos da região afrotropical, e Young e Duncan ${ }^{25}$ (1994) só utilizaram a relação $\mathrm{P} 5 / \mathrm{P} 3+\mathrm{P} 4$ para a chave de subgêneros e grupos de espécies neotropicais.

Tabela 6- Dimensões (em $\mathrm{mm}$ ) de bombas ejaculadoras e dutos ejaculadores e relações dutos ejaculadores/ bombas de machos de L. intermedias. I. do Brasil, Paraguai,

Argentina e Bolívia, agrupados por região.

\begin{tabular}{|c|c|c|c|c|}
\hline $\begin{array}{l}\text { Dimensões } \\
\text { de bombas } \\
\text { ejaculadoras }\end{array}$ & Mínimo & Máximo & M édia & $\mathrm{N}$ \\
\hline \multicolumn{5}{|l|}{ Regiões } \\
\hline $\mathrm{NE}+\mathrm{ME}$ & 177,3 & 227,7 & 198(b) & 49 \\
\hline $\begin{array}{l}\text { ES } \\
\text { RJ }\end{array}$ & $\begin{array}{l}182,8 \\
176,6\end{array}$ & $\begin{array}{l}233,5 \\
224,8\end{array}$ & $\begin{array}{l}209(a) \\
208(a)\end{array}$ & $\begin{array}{l}47 \\
60\end{array}$ \\
\hline $\mathrm{SL}$ & 180,4 & 241,2 & 213(a) & 34 \\
\hline $\mathrm{GO}+\mathrm{MW}$ & 175,0 & 192,5 & $184(c)$ & 13 \\
\hline SI & 168,0 & 211,3 & $190(c)$ & 76 \\
\hline R. Sul & 157,0 & 203,6 & 184(c) & 17 \\
\hline O utros & 163,4 & 208,4 & $186(c)$ & 36 \\
\hline \multicolumn{5}{|l|}{$\begin{array}{l}\text { Dimensões } \\
\text { de dutos } \\
\text { ejaculadores }\end{array}$} \\
\hline \multicolumn{5}{|l|}{ Regiões } \\
\hline ES & 273,4 & 334,8 & $305,8(a)$ & 47 \\
\hline RJ & 244,9 & 374,4 & $302,2(a)$ & 60 \\
\hline SL & 271,8 & 387,1 & $304,4(a)$ & 34 \\
\hline $\mathrm{GO}+\mathrm{MW}$ & 262,5 & 305,1 & $286,5(b)$ & 13 \\
\hline $\mathrm{SI}$ & 245,1 & 349,9 & $296,5(b)$ & 76 \\
\hline R. Sul & 256,2 & 323,3 & $288,1(b)$ & 17 \\
\hline O utros & 246,2 & 327,6 & $293,1(b)$ & 33 \\
\hline \multicolumn{5}{|c|}{$\begin{array}{l}\text { Relações dutos } \\
\text { ejaculadores/ } \\
\text { bombas }\end{array}$} \\
\hline \multicolumn{5}{|l|}{ Regiões } \\
\hline $\mathrm{NE}+\mathrm{ME}$ & 1,12 & 1,82 & $1,52(a)$ & 47 \\
\hline ES & 1,22 & 1,68 & $1,46(b)$ & 47 \\
\hline RJ & 1,22 & 1,73 & 1,46(b) & 60 \\
\hline $\mathrm{SL}$ & 1,25 & 1,70 & $1,43(b)$ & 34 \\
\hline $\mathrm{GO}+\mathrm{MW}$ & 1,36 & 1,75 & $1,56(a)$ & 13 \\
\hline SI & 1,35 & 1,94 & $1,57(a)$ & 81 \\
\hline R. Sul & 1,36 & 1,74 & $1,57(\mathrm{a})$ & 17 \\
\hline O utros & 1,37 & 1,85 & $1,58(a)$ & 33 \\
\hline $\begin{array}{l}\text { ordeste do Brasil; } \\
\text {; RJ- Rio de Janeirc } \\
\text { este de M inas Gera } \\
\text { á, Santa Catarina } \\
\text { ia; N- número de e } \\
\text { es médios seguidos } \\
\text { ntes entre si. }\end{array}$ & $\begin{array}{l}\text { e Sudeste } \\
\text { al de São } \\
\text { a do Mar } \\
\text { de do Sul } \\
\text { medidos. } \\
\text { diferentes }\end{array}$ & $\begin{array}{l}\text { e de Minas } \\
\text { Paulo; G O } \\
\text { re Interior d } \\
\text {;; O utros- Pa } \\
\text { s. }\end{array}$ & $\begin{array}{l}\text { Gerais; ES- Esp } \\
\text { - Goiás; M W- } \\
\text { le São Paulo; } \\
\text { araguai, Argen } \\
\text { ificativamente }\end{array}$ & $\begin{array}{l}\text { Sírito } \\
\text { O este } \\
\text { R. Sul- } \\
\text { tina e } \\
\end{array}$ \\
\hline
\end{tabular}

Seria interessante avaliar melhor a utilidade da fórmula palpal na taxonomia de espécies de flebotomíneos, verificando se a variabilidade observada no presente estudo também ocorre em outras espécies, especialmente as afrotropicais. Se esta variabilidade ocorrer, a utilização da fórmula ficará inviabilizada. Sinton ${ }^{22}$ (1927) propôs o uso da relação entre o comprimento do primeiro artículo e os dos outros artículos, e Pessôa e Barretto ${ }^{18}$ (1948) consideraram esta relação melhor que a fórmula palpal. Entretanto, pela imprecisão na delimitação da extremidade distal do primeiro artículo, esta relação é menos conveniente que a fórmula palpal.

Em ambas as espécies, o labro e os palpos, que são estruturas intimamente associadas, foram maiores nas fêmeas, enquanto o terceiro artículo antenal e a relação antenômerolll/labro foram maiores nos machos. Os carboidratos, consumidos diretamente de vegetais ${ }^{17,20}$ ou de excreções de afídeos ${ }^{11}$, certamente são bem mais acessíveis que o sangue de hospedeiros, o que deve condicionar a diferença positiva no comprimento do labro das fêmeas, em relação aos machos. O maior valor da relação entre o antenômero III e o labro nos machos é mais ligado ao maior comprimento do labro das fêmeas que a diferenças no comprimento do antenômero.

$\mathrm{Na}$ maioria das vezes, as medidas alares foram maiores nas fêmeas, como seria de esperar pelas dimensões mais avantajadas destas. No entanto, a relação comprimento/largura máxima foi maior nos machos, o que está de acordo com a análise de medidas de Phlebotomus perniciosus ${ }^{21}$ e da maioria de outras 17 espécies de Lutzomyia, escolhidas aleatoriamente ${ }^{13}$.

$\mathrm{O}$ fato de flebotomíneos machos mostrarem tendência a enxameamento ${ }^{3,15,16}$ e a comportamento "lek" que é o domínio de território simbólico pelos machos, para demonstrar às fêmeas capacidade de competição ${ }^{23}$, talvez tenha relação com maior agilidade dos machos e com a forma das asas, o que deve ser melhor estudado. Apesar de ter sido observado que culicídeos machos se deslocam menos que as fêmeas ${ }^{19}$, Johnson ${ }^{9}$ (1969) concluiu que as distâncias percorridas por insetos de ambos os sexos de Culicidae, Ceratopogonidae e Simuliidae variam de acordo com a espécie. O estudo de Killick-Kendrick e col. ${ }^{10}$ (1984) sugeriu que os machos de $P$. ariasi deslocam-se menos que as fêmeas. No entanto, os machos de flebotomíneos foram predominantes nas amostras, respectivamente, de recapturas em floresta $^{4}$ e em gado bovino ${ }^{12}$. Alexander ${ }^{2}$ (1987) não encontrou diferenças significativas entre os sexos e Dye e col. ${ }^{6}$ (1991) observaram serem os machos de 
L. longipalpis mais ativos em abrigos de animais domésticos. Em conclusão, os conhecimentos sobre a capacidade de vôo de flebotomíneos são ainda insuficientes para tirar conclusões gerais.

Seria interessante obter fórmulas que relacionassem o comprimento da asa em flebotomíneos com o peso seco e com outras características biológicas, eventualmente de importância na transmissão de patógenos. Christophers ${ }^{5}$ (1960) desenvolveu fórmula para relacionar o comprimento da asa de Aedes aegypti, elevado ao cubo, e o seu peso seco.

As diferenças entre os dois sexos, para os índices alares $(\mathbf{a} / \mathbf{b})$ e a relação entre $\mathbf{d}$ e o comprimento da asa, ressaltam o risco de erro ao usar características das asas para a associação entre os sexos de flebotomíneos pouco conhecidos. Pessôa e Barretto ${ }^{18}$ (1948) consideraram que as medidas alares seriam de pouca utilidade, por sua grande variabilidade. Seria interessante a análise da genética da determinação dos comprimentos das veias e suas relações com o comprimento das asas.

As diferenças nas proporções entre fêmeas com os diferentes tipos de cabeças de espermatecas, nas duas espécies, indica que, apesar de poderem ocorrer vários tipos em ambas, é muito improvável o encontro de espermatecas com cabeças complexas em L. neivai, o que auxilia na identificação das espécies, além das características citadas ${ }^{13}$, como o número de anéis das espermatecas, o comprimento do duto comum e as proporções entre este e os dos dutos individuais.

As diferenças significativas entre os comprimentos das bombas, dos dutos e entre as relações duto/ bomba indicam que essas medidas podem ser úteis para a identificação dos machos. No entanto, sua grande variação e a intersecção dos campos de ambas as espécies diminuem o seu valor para a identificação específica dos machos, ainda difícil e necessitando de outros estudos.

Os comprimentos dos dutos ejaculadores dos machos, em ambas as espécies, foram muito maiores que os comprimentos médios da soma dos dutos comuns e dos individuais das fêmeas. A proporção entre aquele e esta soma, num casal de $L$. neivai montado em cópula, de praticamente 3 vezes (335: $112,5 \mathrm{~mm}$ ), contraria a observação de que tais comprimentos seriam comparáveis em insetos co-específicos $^{24}$. Devem ser feitas comparações em várias espécies, para melhor avaliar esta relação. Hertig ${ }^{7}$ (1949) observou que a parte dos dutos que sofria extrusão era variável, raramente ultrapassando o comprimento dos dutos das fêmeas. As larguras dos dutos de ambos os sexos seriam provavelmente mais similares e úteis para a associação entre os sexos.

\section{AGRADECIMENTOS}

Aos Drs. U. Teodoro da Universidade Estadual de Maringá; H. Taniguchi do Instituto Adolfo Lutz; A. Falqueto da Universidade Federal do Espírito Santo; M. H. Oliveira da Universidade Federal Rural de Pernambuco; S. Oliveira, G. M. Aguiar, M. B. Souza e N. Souza do Instituto Oswaldo Cruz; F. Le Pont do "Office de la Recherche Scientific Technique Outre-Mer, O. D. Salomón do Instituto de Pesquisa Enfermedad de Chagas Fatála Cháben; R. P. Brazil e A. L. Falcão do Centro de Pesquisas René Rachou e R. P. Moraes do Instituto Biológico, pelo auxílio na coleta de insetos e cessão de exemplares e o primeiro também pelas sugestões ao texto; ao Dr. José Sebastião Cunha Fernandes da Universidade Federal do Paraná, pelo auxílio na parte estatística.

\section{REFERÊNCIAS}

1. ABONNENC, E. Les Phlébotomes de la région Éthiopienne (Diptera, Psychodidae). Mem. ORSTOM, (55) 1972.

2. ALEXANDER, J.B. Dispersal of phlebotomine sand flies (Diptera: Psychodidae) in a Colombian coffe plantation. $J$. Med. Entomol., 24:552-8, 1987.

3. ALEXANDER, B. et al. Dispersal of Phlebotomine sand flies (Diptera: Psychodidae) in a Colombian focus of Leishmania (Viannia) braziliensis. Mem. Inst. Oswaldo Cruz, 87:397-403, 1992.

4. CHANIOTIS, B. N. et al. Horizontal and vertical movements of Phlebotomine sandflies in a Panamanian rain forest. J. Med. Entomol., 11:369-75, 1974.
5. christophers, S.R. Aëdes aegypti (L.) the yellow fever mosquito. Cambridge, University Press, 1960.

6. DYE, C. et al. Communication among phlebotomine sandflies: a field study of domesticated Lutzomyia longipalpis populations in Amazonian Brazil. Animal Behav, 42:183-92, 1991.

7. HERTIG, M. The genital filaments of Phlebotomus during copulation. Proc. Ent. Soc. Washington, 51:286-8, 1949.

8. JARVIS, E. K. et al. Laboratory observations on mating and leklike aggregations in Lutzomyia longipalpis (Diptera:Psychodidae). J. Med. Entomol., 29:171-7, 1992. 
9. JOHNSON, C. G. Migration and dispersal of insects by flight. London, Methuen \& Co. Ltd., 1969.

10. KILLICK-KENDRICK, R. et al. Ecology of leishmaniasis in the south of France: dispersal of Phlebotomus ariasi as a factor in the spread of visceral leishmaniasis in the Cevennes. Ann. Parasit. Hum. Comp., 59:555-72, 1984.

11. KILLICK-KENDRICK, R. et al. Honeydew of aphids as a source of sugar for Phlebotomus ariasi. Med. Vet. Entomol., 1:297-302, 1987.

12. LANE, R. P. et al. Antropophagy and aggregation behavior of the sandfly Phlebotomus argentipes in Sri Lanka. Med. Vet. Entomol., 4:79-88, 1990.

13. MARCONDES, C. B. A redescription of Lutzomyia (Nyssomyia) intermedia (Lutz \& Neiva, 1912), and resurrection of L. neivai (Pinto, 1926) (Diptera, Psychodidae, Phlebotominae). Mem. Inst. Oswaldo Cruz, 91:457-62, 1996.

14. MARCONDES, C.B. et al. Distribuição geográfica de flebotomíneos do complexo Lutzomyia intermedia (Lutz \& Neiva, 1912) (Diptera, Psychodidae). Rev. Soc. Bras. Med. Trop., 31:51-8, 1998.

15. Memmott, J. Patterns of sandfly distribution in tropical forest: a causal hypothesis. Med. Vet. Entomol., 6:188-94, 1992.

16. MILES, C.T. et al. Mating aggregations of male Lutzomyia sandflies at human hosts in Panama. Trans. R. Soc. Trop. Med. Hyg., 70:531-2, 1976.

17. PARROT, L. M. Phlébotomes et végétation. Bull. Soc. Path. Exot., 28:960-3, 1935.
18. PESSÔA, S. B. \& BARRETO, M.P. Leishmaniose tegumentar americana. Rio de Janeiro, Ministério da Educação e Cultura, Imprensa Nacional, 1948.

19. RUSSELL, P.F. et al. Some experiments on flight range of Anopheles culicifacies. J. Exp. Zool., 97:135-63, 1944.

20. SCHLEIN, Y. et al. Leishmaniasis in the Jordan Valley. IV. Attraction of Phlebotomus papatasi (Diptera:Psychodidae) to plants in the field. J. Med. Entomol., 24:87-90, 1987.

21. SILANS, L. N. M. P. Étude de la morphométrie alaire intra-spécifique et entre populations chez Phlebotomus (Larroussius) perniciosus Newstead, 1911 (Diptera: Psychodidae) par un système de mesure semi-automatique. Montpellier, 1994. [Diplôme études Approfondies, Parasitologie- Lab. Écol. Méd. Pathol. Paras., Fac. Médécine Montpellier].

22. SINTON, J.A. Notes on some Indian species of the genus Phlebotomus. XVIII. Miscellaneous notes. Ind. J. Med. Res., 14:947-53, 1927.

23. THORNHILL, R. et al. The evolution of insect mating systems. Cambridge, Harvard University Press, 1983.

24. WILLIAMS, P. A female sand fly (Diptera: PsychodidaePhlebotominae) similar to Brumptomyia spinosipes (Floch \& Abonnenc, 1943). Mem. Inst. Oswaldo Cruz 83:343-6, 1988

25. YOUNG, D. G. \& DUNCAN, M. A. Guide to the identification and geographic distribution of Lutzomyia sand flies in Mexico, the West Indies, Central and South America (Diptera: Psychodidae). Mem. Am. Entomol. Inst., 54:1-881, 1994 